\title{
Spectral Investigations of UV Ceti-Type Flare Stars and Search for New Variables of this Type Carried out at Crimea
}

\author{
R. E. GERSHBERG and N. I. SHAKHOVSKAYA (Crimea)
}

(Read by E. SCHÖFFEL)

During last years, after Budapest Colloquium, flare star investigations have been continued at the Crimean Astrophysical Observatory. The purpose of the investigations was to specify spectral features of flares, to research quantitatively spectra of the stars in quiet state and to search for new variables of this type.

\section{Spectral investigations of flares of UV Cet-type stars}

1. In January 1969 we have carried out simultaneous photometric and spectral patrol of flare star YZ CMi using method described earlier (1). Photoelectric patrol of star brightness was realized at meniscus $64 \mathrm{~cm}$ telescope in B-band as a part of international programme of photometric observations of this star (2). Image tube spectrograph at $2,6 \mathrm{~m}$ Shajn reflector allows us to obtain YZ CMi spectrum from 3500 to $7000 \AA$ with $340 \AA / \mathrm{mm}$ dispersion and $0,4 \mathrm{~mm}$ widening during $1^{\mathrm{m}}-2^{\mathrm{m}}$ exposure. Three stage image tube made by Prof. M. M. BUTSLOV (3) was used in spectrograph. During 6 nights patrol we have registered 5 flares. Their light curves are shown in Fig. 1; numbered horizontal segments under light curves indicate time intervals of spectral exposures. All spectrograms of flares and about two dozens spectrograms of the star at quiet state were reduced spectrophotometrically. As a result we have found the following features of the YZ CMi flares (4).

The molecular band systems that are characteristic in visual and red regions of red dwarf star spectra remain during the flares; it is therefore an evidence that the quick processes at stellar surface are local but not global ones. Hydrogen and calcium emission lines seen well at quiet state spectra strengthen up to several times when total brightness of the star increases up to om $2-0 m 5$. Firstly hydrogen lines strengthen sharply. And calcium lines' decrease takes much more time, perhaps many hours after a flare has ceased to be observable photometrically. Balmer decrement of flares is very slope and evidences obtained on existing of the inverse ratios of intensities: $\mathrm{I}_{\mathrm{H}_{\beta}}<\mathrm{I}_{\mathrm{H}_{y}}<\mathrm{I}_{\mathrm{H}_{\delta}}$.

Spectral features of the YZCMi flares listed above are similar to flare features of other UV Cet-type stars but many hours Call radiation is a qualitatively new fact.

2. In March 1970 we have obtained two spectrograms of the strong flare of AD Leo; the amplitude and the duration of this flare are similar to ones of the flare of the same star on 18.5.65 (1). The light curve of the flare on 2.3.70 and time of spectrographying are shown in Fig. 2. Spectral observations were carried out at Shajn reflector using the grating spectrograph SP-72 with $150 \AA / \mathrm{mm}$ dispersion and the emulsion A-700.

$\mathrm{AD}$ Leo registrograms obtained during the flare on 2.3.70 and in quiet state on 27.4.70 are presented in Fig. 3. On the second date photoelectric patrol of star brightness was carried out too. Comparison of the spectrograms shows an effect of flare continuum radiation. On the first spectrogram this radiation veils the absorption line $\lambda 4227 \AA \mathrm{Call}$ and strong $\mathrm{TiO}$ absorption bands $\lambda 4761 \AA$ and $\lambda 5862 \AA$ totally and in region $\lambda<4200 \AA$ almost the whole radiation registered is due to the flare. Balmer emission continuum certainly observed transits into quasicontinuum formed by overlapping of Balmer series high members. On the second spectrogram the flare continuum radiation is seen less clear. Balmer emission discontinuity $D_{B}$ evaluated according to the magnitude $\log \mathrm{I}_{\lambda 3800}-I_{\lambda \text { s800 }}+$ is equal to 0.15 ; this value is somewhat less than $D_{B}$ found spectroscopically by KUNKEL (5) and nearer to $D_{B}$ which was evaluated by CHUGAINOV (6) by photoelectric measurements of flares in three narrow spectral bands. If the discontinuity observed is interpreted within the framework of pure recombination radiation hypothesis and $D_{B}<0.2$ then electron temperature of gas must exceed 

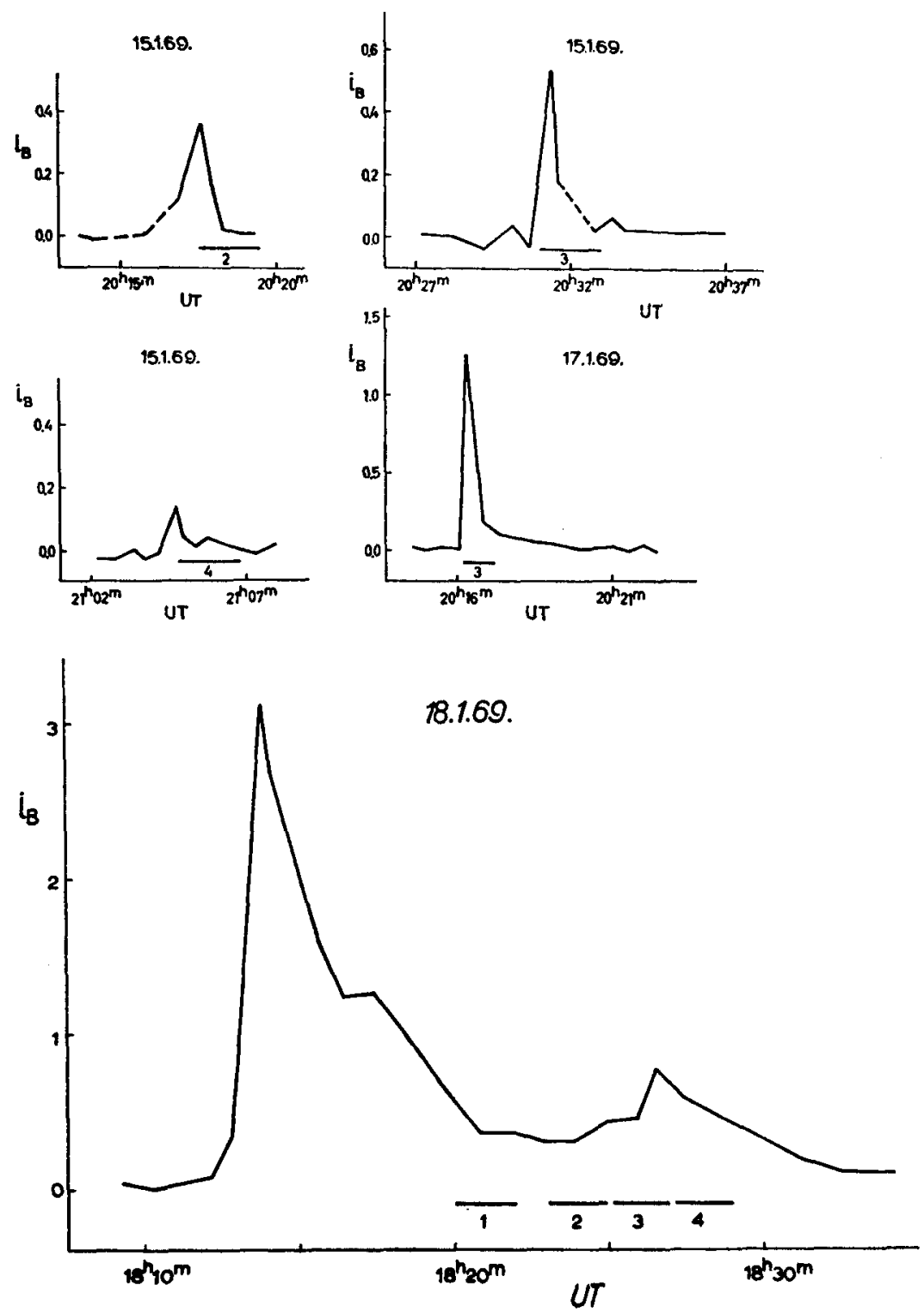

Fig. 1: Light curves of the YZ CMi flares. 


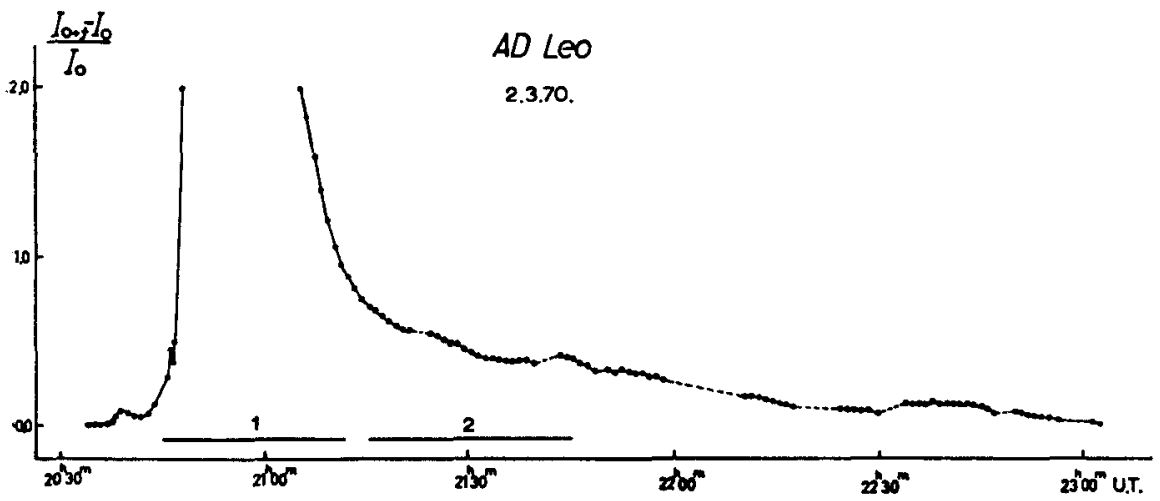

Pig. 2: The light curve of the AD Leo flare on 2.3.70.

$40000^{\circ} \mathrm{K}$. The discontinuity increasing during flare decay found by CHUGAINOV (6) can be related to a quick cooling of gas.

Results of spectrophotometric reduction of emission lines are given in Table 1 and in Fig. 4. Equivalent widths and relative intensities of the lines during the AD Leo flare on 2.3.70 are close to corresponding values in spectra of other strong flares of UV Cet-type stars. But using the spectra of this flare we could first evaluate line widths of several emission lines simultaneously. Table 1 shows that the ratio $\Delta \lambda / \lambda$ varies noticeably from line to line, therefore Doppler broadening is not the main factor in line profile forming. Fig. 4 gives line profiles measured. Resolution of spectra obtained is insufficient to allow profile investigation in detail because emission line half-widths measured in flare spectra are only twice as large as instrumental profile half-width. However two important features are seen certainly in Fig. 4. There is a red asymmetry on the first flare spectrogram: the long-wave wings of the lines are more extended from the points of maximum intensity than the short-wave wings are extended. On the second spectrogram of the flare lines profiles are quite symmetrical but profile tops are flatter and one can suspect a small intensity decreasing exists at some centers of profiles. Emission line width evaluations noted above exclude an interpretation of profile asymmetry within the framework of simple Doppler effect. As it is known similar asymmetric profiles are observed in solar chromospheric flares too (7) and line asymmetry is characteristic feature just for the beginning of solar flare development $(8,9)$. It seems that in order to interpret the short-lived asymmetry discovered one ought to attract the non-coherent scattering theory for optical thick medium with velocity gradient. Detail description of spectra of the AD Leo flare an 2.3.70 is given in (10).

Table 1

\begin{tabular}{|c|c|c|c|c|c|c|c|c|c|}
\hline & & & $\mathrm{H}_{\alpha}$ & $\mathrm{H}_{B}$ & $\mathrm{H}_{y}$ & $\mathrm{H}_{\delta}$ & $\mathrm{H}+\mathrm{H}_{\varepsilon}$ & $\mathrm{K}$ & $\overline{\mathrm{H}_{8}}$ \\
\hline \multirow{3}{*}{$\begin{array}{l}\text { Equivalent widths } \\
\text { of emission lines }(\AA)\end{array}$} & 2.3 .70 & 1 & 12 & & 16 & 13 & 14 & 4.9 & 12 \\
\hline & 2.3 .70 & 2 & 14 & & 51 & 35 & & & \\
\hline & 27.4 .70 & & 4.3 & 4.2 & 6.5 & 5.0 & 16 & 11 & 5.9 \\
\hline \multirow{3}{*}{$\begin{array}{l}\text { Relative intensities } \\
\text { of emission lines }\end{array}$} & 2. 3.70 & 1 & 1.0 & $.91:$ & .80 & .63 & .66 & .25 & $.57:$ \\
\hline & 2.3 .70 & 2 & 1.0 & & .76 & .47 & .58 & .27 & $.31:$ \\
\hline & 27.4 .70 & & 1.0 & .74 & .52 & .29 & .52 & .30 & $.16:$ \\
\hline \multirow{3}{*}{$\begin{array}{l}\text { Widths of emission } \\
\text { lines measured at } \\
\text { half-intensity level }(\AA)\end{array}$} & 2.3 .70 & 1 & 10 & & 10 & 13 & 10 & & \\
\hline & 2.3 .70 & 2 & 12 & & 12 & 13 & 13 & & \\
\hline & 27.4 .70 & 7 & width & & $\operatorname{rmin}$ & by th & e instru & to & file \\
\hline
\end{tabular}




\section{AD Leo}

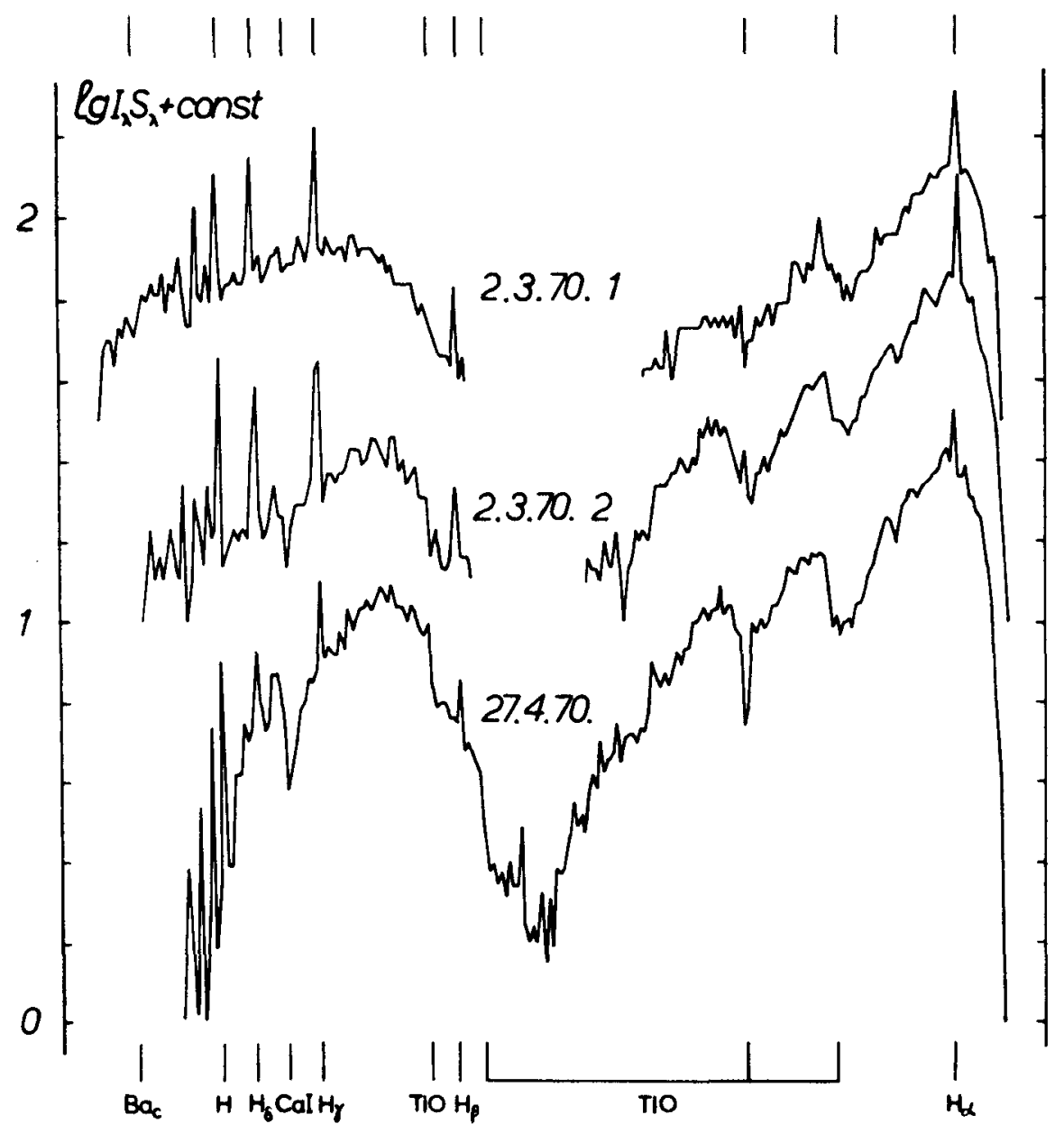

Fig. 3: Registrograms of AD Leo spectra obtained during the flare on 2.3.70 and in quiet state. 

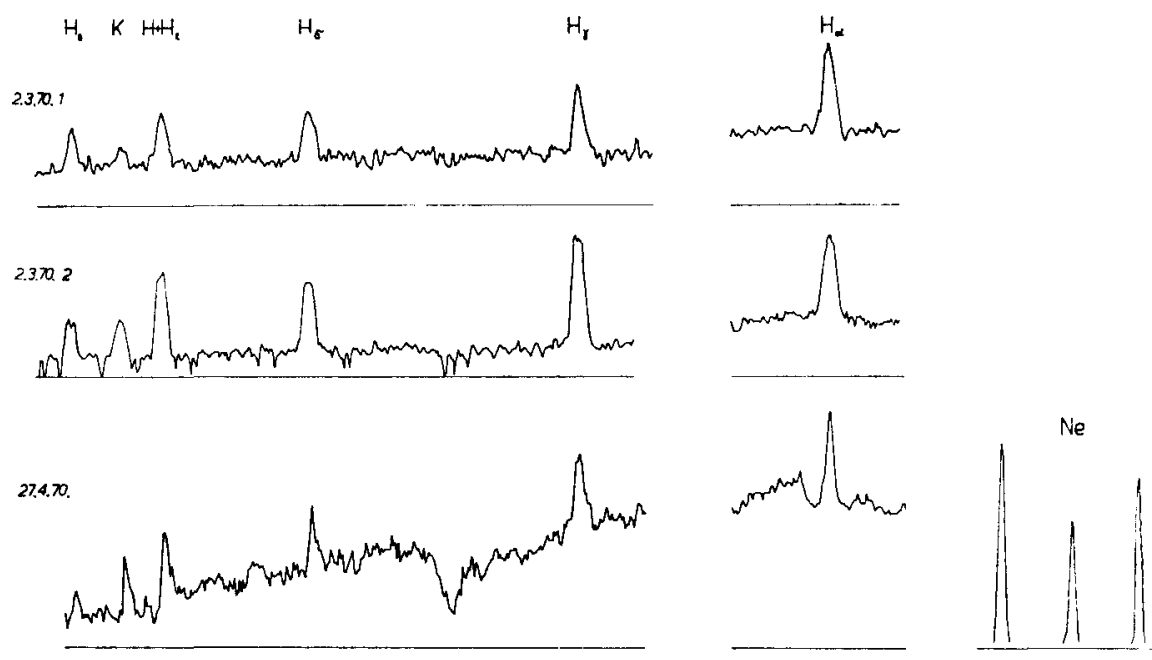

Fig. 4: Emission line profiles in AD Leo spectra obtained during the flare on 2.3.70 and in quiet state.

3. We have obtained a spectrum of the EV Lac flare on 12.7.66 using the same spectrograph SP-72 and Shajn reflector. Results of photometric reduction of this spectrum and light curve of the flare are reported in (11). Balmer emission decrement theory for UV Cet-type star flares is also considered in that paper.

As it is known hydrogen line intensity ratios in flare spectra have been first determined by KUNKEL (5) and in order to explain very slope and inverse Balmer decrement found KUNKEL has carried out calculation of the emission from optical thick medium in $\mathrm{H}_{a}$ assuming scattering in lines to be coherent. He has succeeded to present the decrement observed but it is hard to expect that this model to be adequate to real flare conditions. Therefore KAPLAN (11) has calculated Balmer decrement for medium with velocity gradient accounting noncoherent scattering in lines and supposing a large optical thickness in frequencies of several first series members. Calculation has shown that in such a model a large set of slope and inverse decrements can be obtained by variation of the only one parameter, namely, an emission measure of radiating gas. In particular this model gives a possibility to represent both slope decrement observed in spectrum of the EV Lac flare on 12.7.66 and inverse decrement in spectra of the flare of the same star which were measured by KUNKEL (5) on 11.12.65. If a characteristic size of a flare is about $10^{9} \mathrm{~cm}\left(\sim 0.07 R_{*}\right)$ then emission measures which correspond to observed decrement in accordance to theoretical model considered lead to flare electron density evaluations as large as $10^{12}-3 \times 10^{13} \mathrm{~cm}^{-3}$.

Another variant of Balmer decrement theory for medium with velocity gradient and noncoherent scattering has been considered in detail by BOYARCHUK (12); in this variant the shock interactions of particles are not taken into account but specific values for ionizing radiation flux and for escape probabilities of scattering photons from the gas are used. Theory (12) gives a good representation for Balmer decrement of the EV Lac flare on 12.7.66 if the escape probability for $\mathrm{H}_{\beta}$-quantum is about $10^{-3}$. The same theoretical model predicts the ratio of $\mathrm{H}_{\beta}$-line intensity to adjacent continuum emission intensity which is close to this ratio measured by CHUGAINOV during photoelectric observations of the flare in two narrow interference filters. 


\section{Spectral investigations of UV Cet-type stars in quiet state}

As a rule strong emission hydrogen and calcium lines are seen in UV Cet type star spectra obtained in quiet state of a flare star. This emission is interpreted usually as an evidence for strong dhromosphere existing. Discovery of power coronas on flare stars is an additional confirmation of the idea that non-radiative energy flux is very important in atmospheres of these dwarf stars. Therefore quiet state spectrograms which have been obtained at Shajn reflector during last years for flare stars have been analysed on the assumption that permanent emission observed has a chromospheric nature.

1. As it follows from WILSON's paper (13), ionization and excitation degrees of matter and inner motion velocities in the EV Lac chromosphere are close to corresponding values in solar chromosphere. Neglecting of known temperature and ionization gradients in solar chromosphere and of deviations from spheric symmetry in its structure we suppose that for the first approximation solar and stellar chromospheres may be described as an isothermal spherically symmetric envelope with barometric density distribution. In such a model Balmer emission decrement and absolute enission line luminosity are determined only by the radiating gas mass in a column at a line of sight.

Comparison of Balmer decrements in solar chromosphere and in quiet dhromospheres of $\mathrm{AD}$ Leo and EV Lac are given in Fig. 5. Solar decrements have been obtained during eclipse
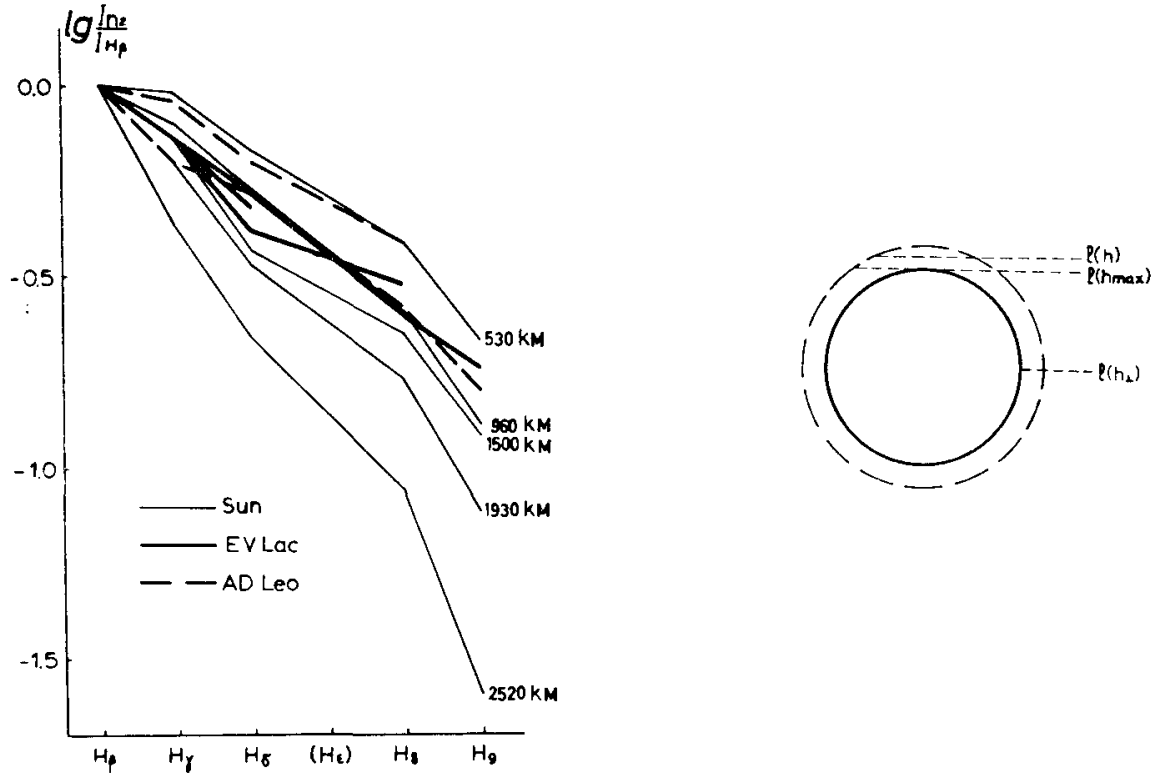

Fig. 5: Comparison of Balmer decrement of solar chromosphere with ones of flare stars.

when the edge of the Moon has located at various heights above the solar limb (14); the heights are indicated near the corresponding lines. As it follows from the plot Balmer decrement of flare stars - observed only averaged over a stellar disc - is close to solar chromosphere Balmer decrement if the line of sight runs at height $500-1500 \mathrm{~km}$ above the level of photosphere. It can be shown that the gaseous mass in column at the line of sight which runs through a barometric envelope is in average twice as large as the gaseous mass in column at the line of sight which runs through the center of the stellar disc. Let us consider the line of sight that runs at the height $h$ above the bottom of a sperical and barometric envelope which has a inner radius $R_{*}$ and equivalent thickness $h_{0}$. Then the ratio of the gaseous mass at this line of sight to the gaseous mass at the line of sight which runs through the center of the stellar disc is determined as 


$$
\frac{\mathrm{m}(\mathrm{h})}{\mathrm{m}_{0}}=2.5\left(\frac{\mathrm{R}_{*}}{\mathrm{~h}_{0}}\right)^{1 / 2} \mathrm{e}^{-\mathrm{h} / \mathrm{h}_{0}}
$$

Using this relation, known values $R_{*}$, and $h_{0}$ for the Sun and the correspondence observed between stellar and solar decrements one may conclude that the number of hydrogen atoms at the second energetic level above $1 \mathrm{~cm}^{2}$ photospheric surface of a flare star is about 10 times as large as this number of atoms above $1 \mathrm{~cm}^{2}$ solar photospheric surface. Then, equivalent thickness of barometric envelopes with the same temperature are determined as

$$
h_{0} \propto \frac{M_{*}}{R_{*}^{2}}
$$

where $M_{*}$ is a stellar mass. Ratios $M_{*} / R_{*}{ }^{2}$ for UV Cet-type stars are 3-10 times less than for the Sun, and stellar chromospheres must be correspondingly less extended compared to solar chromosphere. Consequently, densities of hydrogen atoms $\mathrm{N}_{2}$ in stellar chromospheres are dozens time more compared to density in corresponding levels of solar chromosphere.

Similar results can be obtained from the analysis of absolute luminosity of flare star chromospheres. Using equivalent widths of hydrogen emission lines which have been measured in quiet state spectra of EV Lac, AD Leo, UV Cet, Wolf 630 and YZ CMi, we have calculated surface brightnesses of stellar dromospheres in the lines; the result is $(2-4) \times 10^{6} \mathrm{erg} / \mathrm{cm}^{2} \mathrm{sec}$ that is about twice as large as surface brightness of solar chromosphere. According to Balmer decrement observed there is a self-absorption in lines; therefore chromospheric emission in hydrogen lines does not correspond to the linear part of a curve of growth. But this emission cannot correspond to the upper part of the curve of growth too because $\mathrm{H}_{a}$-line profiles observed do not show strong wings (see below) which are characteristic for this part of the curve of growth. Hence, an intermediate part of the curve of growth remains. Outpur of energy here is proportional to $\left(\ln \mathrm{N}_{2}\right)^{1 / 2}$; therefore, two-fold difference in absolute surface brightness corresponds to 50 -fold difference in number of atoms at the line of sight.

Although the absolute surface brightnesses of stellar and solar chromospheres in hydrogen lines are similar, however non-radiative energy flux density which runs through flare star photospheres must be mudh more than that at the Sun because in upper levels of stellar photospheres gaseous density is much more and gaseous temperature is much less than that in corresponding levels of solar atmosphere.

Higher gaseous density in a chromosphere can lead to existing of a more dense corona than solar one. Preliminary coronal density estimates obtained during the YZ CMi flare (15) confirm this supposition.

2. In order to consider preliminarily emission $\mathrm{H}_{\alpha}$-line features in quiet chromospheres of flare stars, we have obtained 8 spectra in red, using the short-focus camera of Shajn reflector coudé spectrograph with $24 \AA / \mathrm{mm}$ dispersion. Emission $\mathrm{H}_{a}$-line profiles obtained from the spectra have a simple bell-like shape without a central depression and wide wings as a rule. Data on spectra obtained and profile widths measured at half-intensity levels are listed in Table 2. Measured half-widths have been corrected for an instrumental profile approximatively and give us estimates of emission half-widths $\Delta \lambda_{1 / 2}$; these values give an upper limit of stellar rotational velocity at chromospheric level $\mathrm{v}_{\mathrm{I}} \sin \mathrm{i}$.

Comparison emission $\mathrm{H}_{a}$ profiles obtained for stellar chromospheres and numerous data on $\mathrm{H}_{\alpha}$ profiles in solar dromosphere allows to conclude that stellar emission profiles are apparently slightly narrower than ones are in low and middle dhromosphere at the Sun. Stellar emission profiles do not show depression at the center of profiles which is so characteristic for solar chromospheric profiles; however this difference expects to be confirmed by observations with better spectral resolution. On the other hand, it is possible that the difference is real but it is due not to a difference between chromospheres themselves. It may be due to different contributions which photospheric radiation scattered by chromosphere carries in emission $\mathrm{H}_{a}$ profile. At the Sun this contribution is very noticeable and at flare stars it is apparently negligible. 
Table 2

\begin{tabular}{lcccccc}
\hline Stars & Date & $\begin{array}{c}\text { Exposures } \\
\text { (hours) }\end{array}$ & $\begin{array}{c}\text { Half-widths } \\
\text { measured }(0.01 \mathrm{~mm}) \\
\mathrm{H}_{\alpha}\end{array}$ & $\begin{array}{c}\Delta \lambda_{1 / 2} \\
\text { instrum. }\end{array}$ & $\begin{array}{c}\mathrm{v}_{\mathrm{r}} \sin \mathrm{i} \\
(\mathrm{Am})\end{array}$ & $\begin{array}{l}\mathrm{km} / \mathrm{sec}) \\
\text { YZ CMi }\end{array}$ \\
& 22.2 .69 & 6 & 6.4 & 4.0 & 1.2 & 55 \\
& 6.3 .69 & 3 & 5.6 & 2.2 & 1.2 & 55 \\
& 23.3 .69 & 4 & 5.2 & 2.9 & 1.0 & 46 \\
AD Leo & 26.3 .69 & 3.8 & 6.4 & 2.8 & 1.4 & 62 \\
& 18.5 .68 & 2 & 6.8 & 4.0 & 1.3 & 59 \\
& 2.3 .69 & 2.7 & 5.7 & 2.6 & 1.2 & 54 \\
EV Lac & 6.3 .69 & 1 & 5.3 & 2.2 & 1.1 & 52 \\
& 3.12 .68 & 5 & 6.0 & 3.3 & 1.2 & 54 \\
\hline
\end{tabular}

The results of investigations of flare star quiet chromospheres which are given above shortly are published in paper $(16,17)$.

3. In years 1968-1970 we used Shajn reflector coudé spectrograph for comparative spectrophotometrical analysis of red dwarfs. We have obtained 3 spectrograms of the flare star $\mathrm{AD}$ Leo and 6 spectrograms of Barnard's star that is the known subdwarf with a large proper motion; up to now the attempts to find any flaring of this star are unsucessful. Our spectrograms cover the wave-length interval from $5600 \AA$ to $7000 \AA$ and have 12 and $24 \AA / \mathrm{mm}$ dispersion. After reducing the plates BOYARCHUK (18) has determined relative abundances of $\mathrm{Li}$ and $\mathrm{Ca}$. She has found that in Barnard's star atmosphere the ratio $\mathrm{N}(\mathrm{Li}) / \mathrm{N}(\mathrm{Ca})$ is. 14 times less compared to solar atmosphere. For $\mathrm{AD}$ Leo the upper limit of $\mathrm{Li}$ abundance is only found, this abundance is at least 160 times less than solar one.

\section{Search for new UV Cet-type variables}

At the Crimean Astrophysical Observatory side by side with investigations of the known UV Cet-type flare stars, photometric and spectral observations have been carried out for a set of $d M$ and $d M e$ stars; till the beginning of these observations there were not exhaustiveinformations on flare activity of these M-stars.

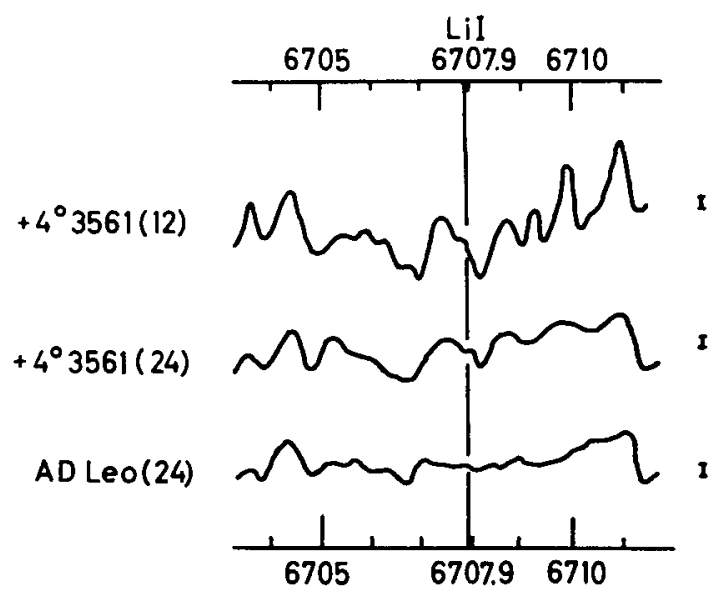

Fig. 6: Registrograms of Barnard's star (= BD $+4^{\circ} 3561$ ) and AD Leo in Li line region. Numbers in brackets are dispersion values. Vertical segments indicate mean fluctuation due to emulsion grains. 


\section{$A C+39^{\circ} 1214-608$}
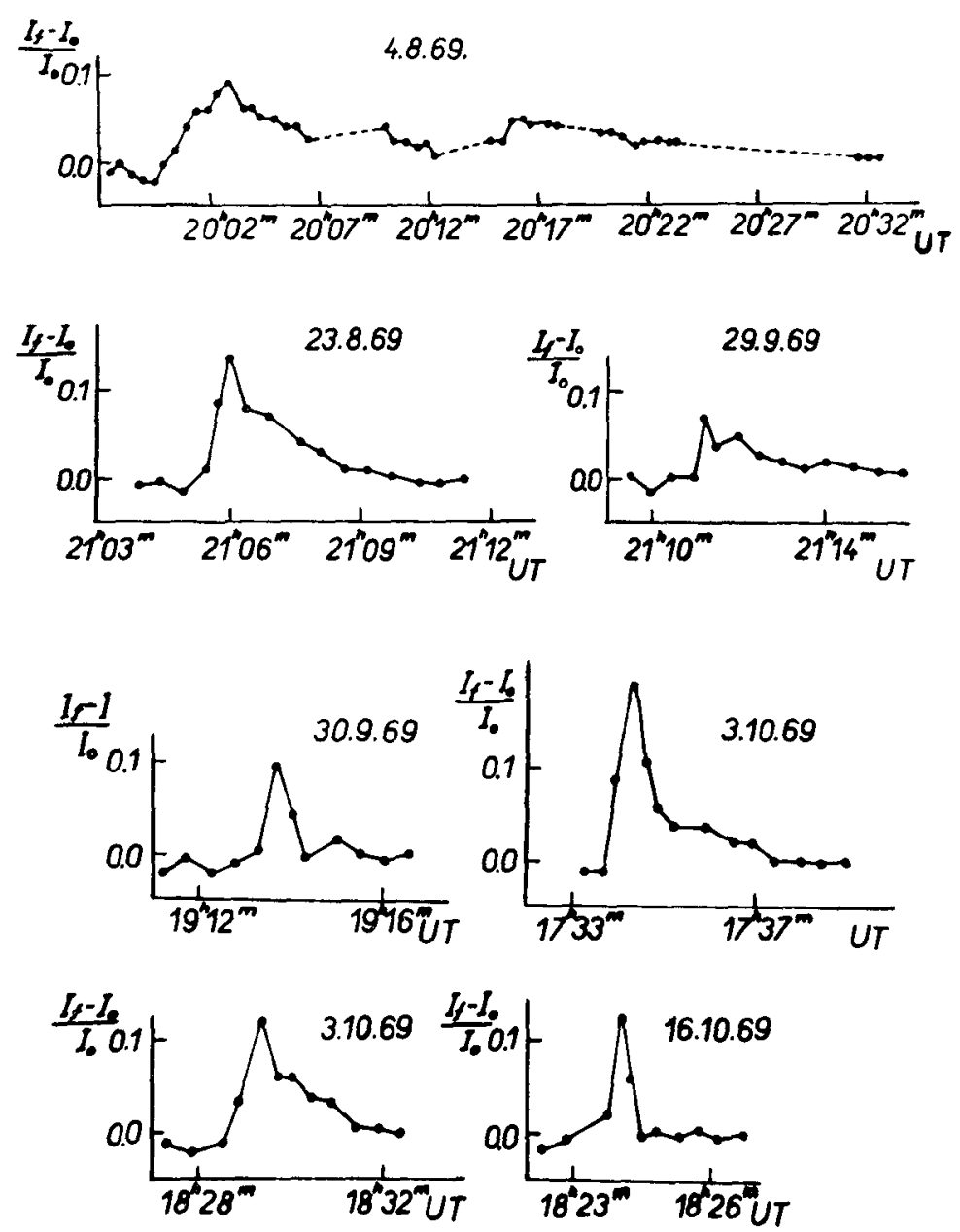

Fig. 7 : Light curves of the $\mathrm{AC}+39^{\circ} 1214-608$ flares. 
All patrol photoelectric observations are carried out at $64 \mathrm{~cm}$ meniscus telescope in B-band. Preliminarly results of the observations according to (19-23) are given in Table 3. In the last column of the Table there are logarithms of ratios $\Sigma \mathrm{P}_{\mathrm{B}} / \mathrm{T}=\int \Delta \mathrm{I}_{\mathrm{B}} \mathrm{dt} / \int \mathrm{I}_{\mathrm{B}}{ }^{0 \mathrm{dt}}$, where $\mathrm{I}_{\mathrm{B}}{ }^{0}$ is a star brightness in quiet state, $\Delta \mathrm{I}_{\mathrm{B}}$ is brightness increase during a flare and integrating is realized over the whole time of brightness patrol. This magnitude is close to KUNKEL's "observed index" and gives the most complete idea on contribution of flares in total stellar luminosity.

From variables listed in Table 3 stars in systems $20 \mathrm{C} 1250$ and $\mathrm{BD}+43^{\circ} 44$ are the most interesting $(19,20)$.

The system $20 \mathrm{C} 1250\left(=\mathrm{AC}+39^{\circ} 1214-608\right)$ was attributed to UV Cet-type variables supposedly by JOY based on emission line variability. We have registered 7 flares, there amplitudes are from omo8 to om 19 . In accordance to shape of light curves and to flare rise and decay time (see Fig. 7) the star must be certainly attributed to UV Cet-type. We have obtained 7 spectrograms of the star in quiet state also. Balmer decrement and calcium line equivalent widths of this star are very similar to that of EV Lac and AD Leo, but hydrogen line equivalent widths of $20 \mathrm{C} 1250$ is about twice less than of stars named. The line intensity variability that is not connected to a flare during spectrographying is confirmed. A cause of such variability may be a slow decay of line radiation after photometric phases of a flare that can have a maximum long before the spectrographying and/or season variations of a total power of a stellar chromosphere. The star $20 \mathrm{C} 1250$ is bright enough $\left(\mathrm{mB}_{\mathrm{B}}=11 \mathrm{~m} 6\right)$ and has a position that is suitable for observers in northern hemisphere; therefore it is an appropriate object for flare star investigators.

Table 3

\begin{tabular}{ccccr}
\hline $\begin{array}{c}\text { Star number in } \\
\text { accordance to (24) }\end{array}$ & Name of star & $\begin{array}{c}\text { Duration of photo- } \\
\text { electric patrol (hours) }\end{array}$ & $\begin{array}{c}\text { Number of flares } \\
\text { recorded }\end{array}$ & log $\frac{\Sigma P_{\mathrm{B}}}{\mathrm{T}}$ \\
\hline $15 \mathrm{~A}$ & $\mathrm{BD}+43^{\circ} 44 \mathrm{~A}$ & 40.3 & 3 & -5.8 \\
$15 \mathrm{~B}$ & $\mathrm{BD}+43^{\circ} 44 \mathrm{~B}$ & 46.1 & 16 & -3.0 \\
424 & $\mathrm{SZ} \mathrm{UMa}$ & 28.9 & 1 & -3.7 \\
494 & $\mathrm{BD}+13^{\circ} 2618$ & 72.7 & 4 & -2.8 \\
569 & $\mathrm{BD}+16^{\circ} 2708$ & 30.1 & $1 ?$ & $<-4.6$ \\
$\left.644 \mathrm{AB}^{*}\right)$ & $\mathrm{V} 1054 \mathrm{Oph}=$ Wolf 630 & 23.4 & 1 & -2.9 \\
$\left.725 \mathrm{AB}^{*}\right)$ & $\mathrm{BD}+59^{\circ} 1915$ & 23.2 & 17 & $<-4.8$ \\
735 & $\mathrm{AC}+8^{\circ} 142-393$ & 26.6 & 5 & -2.7 \\
$\left.815 \mathrm{AB}^{*}\right)$ & $20 \mathrm{C} 1250 \mathrm{AB}=$ & & & -3.2 \\
& $=\mathrm{AC}+39^{\circ} 1214-608$ & 47.4 & 7 & $<-4.4$ \\
908 & $\mathrm{BD}+1^{\circ} 4774$ & 44.9 & 0 & \\
\hline
\end{tabular}

*) During brightness patrol components of the binary system were not separated.

JOY has supposed the star $B D+43^{\circ} 44 \mathrm{~B}$ was an UV Cet-type based on the fact that hydrogen emission was seen only on one of several spectrograms. Our patrol observations show photometric flare activity in both components in the system BD $+43^{\circ} 44$ (see Table 3 ). As in previous case, a shape of light curves of flares removes all doubts on a kind of variability, these stars are certainly UV Cet-type variables (see Fig. 8). However, we have not found hydrogen emission lines in spectra of these stars. Then, kinematic and photometric features of the system $B D+43^{\circ} 44$ show definitely that these stars belong to old population (25). Therefore, from the fact that components of $\mathrm{BD}+43^{\circ} 44$ show the flare activity of the UV Cet-type one can draw two important conclusions. Firstly, such kind of activity is inherent feature in stars whose chromospheres can have a wide range of power. Secondly, stars of very different ages can show such an activity. 
Stars V 1054 Oph (= Wolf 630 ) and SZ UMa are also rather old objects, they belong to halo or to old disc population. Therefore, their noticeable flare activity as well as the activity of $\mathrm{BD}+43^{\circ} 44$ contradicts with widespread idea that the flare activity is inherent in young stars only. Note that our spectrograms of SZ UMa also do not show hydrogen and ionized calcium emission lines.

Our spectral observations of red dwarf stars allow to present in quantitative form the known qualitative correlation between hydrogen line intensity in quiet state star spectra and its flare activity level. Such a correlation is given in Fig. 9 where we compare the equivalent widths of emission $\mathrm{H}_{\alpha}$ line with the magnitudes $\log \frac{\Sigma \mathrm{P}_{\mathrm{B}}}{\mathrm{T}}$. (The lower limit of $\mathrm{W}_{\mathrm{H}_{\alpha}}$ certainly registered on our spectrograms is about $1 \AA$.) Comparison visual estimates of calcium line intensities from (26) with our values $\log \Sigma \mathrm{P}_{\mathrm{B}} / \mathrm{T}$ gives similar results, see Fig. 10.

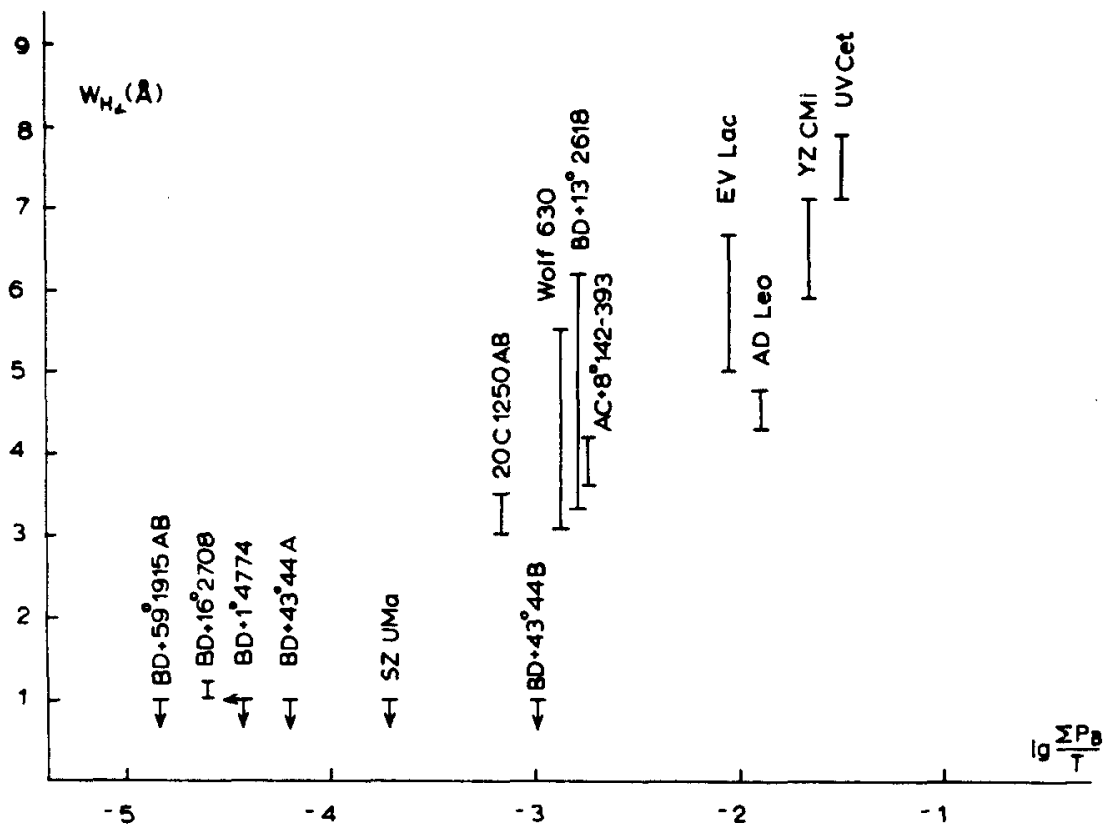

Fig. 9: Correlation between flare activity levels of stars and $\mathrm{H}_{a}$ emission intensities in their quiet state spectra. 

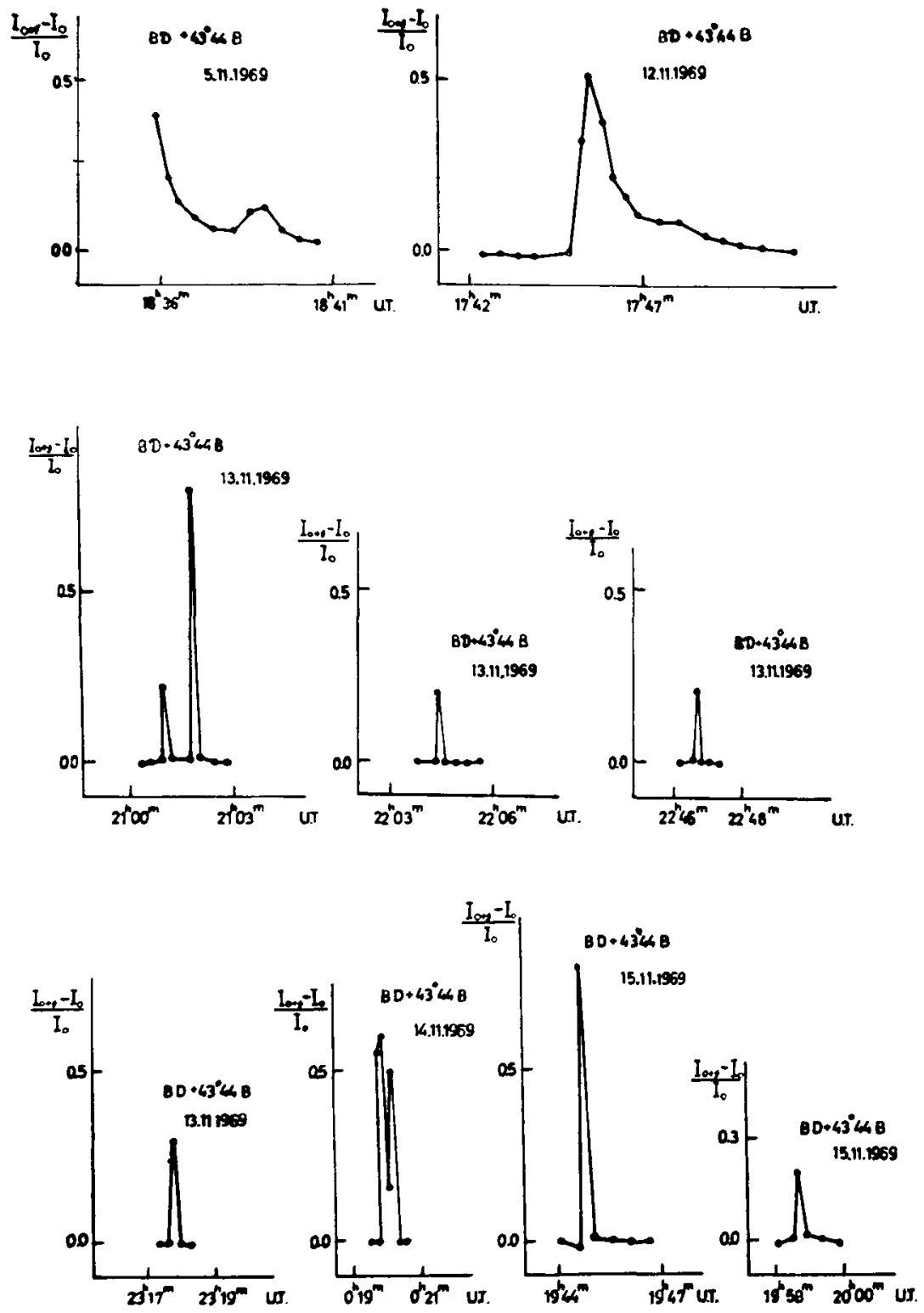
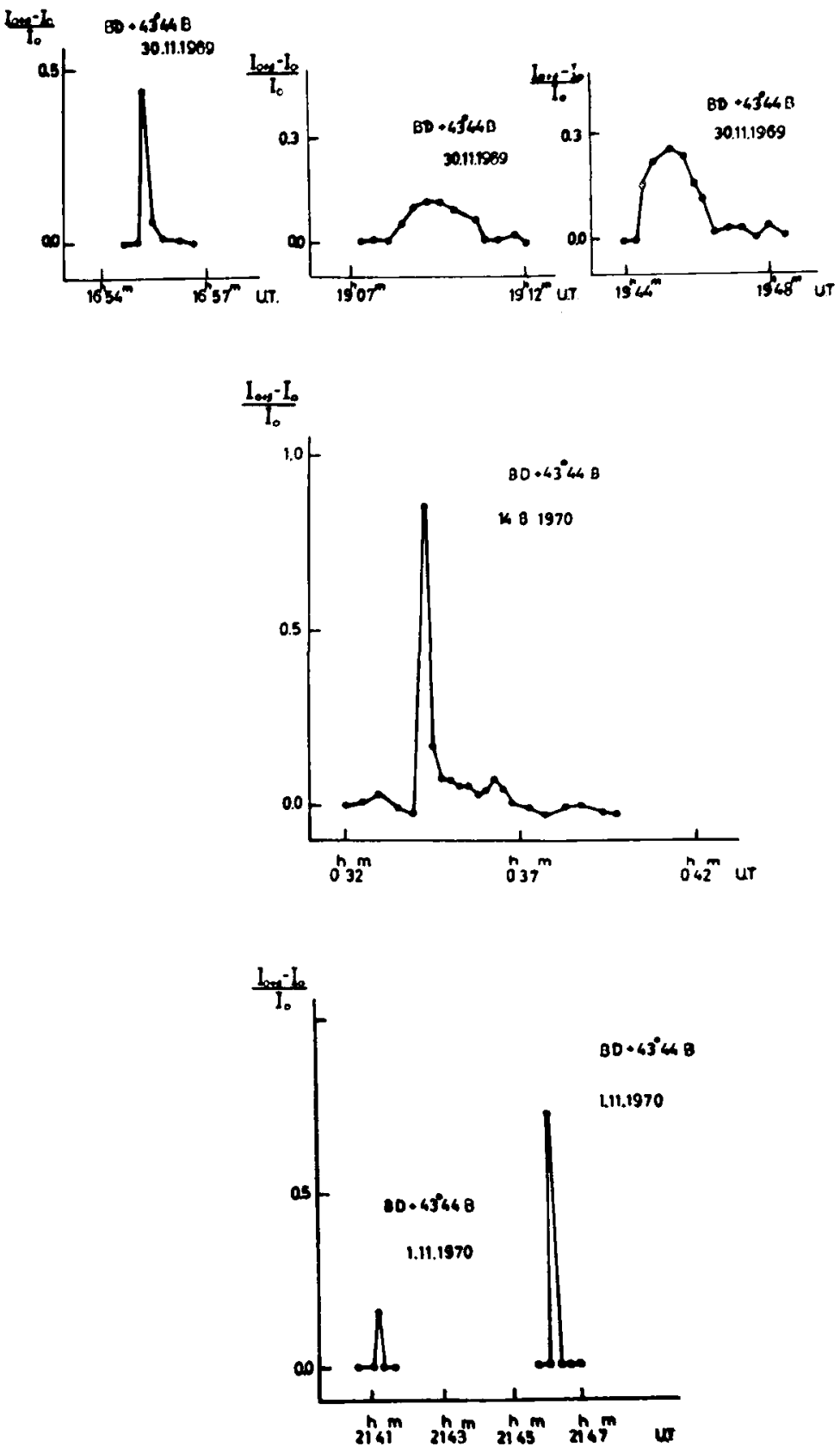

Fig. 8: Light curves of the $\mathrm{BD}+43^{\circ} 44 \mathrm{~B}$ tares.

https://doi.org/10.1017/S0252921100032851 Published online by Cambridge University Press 


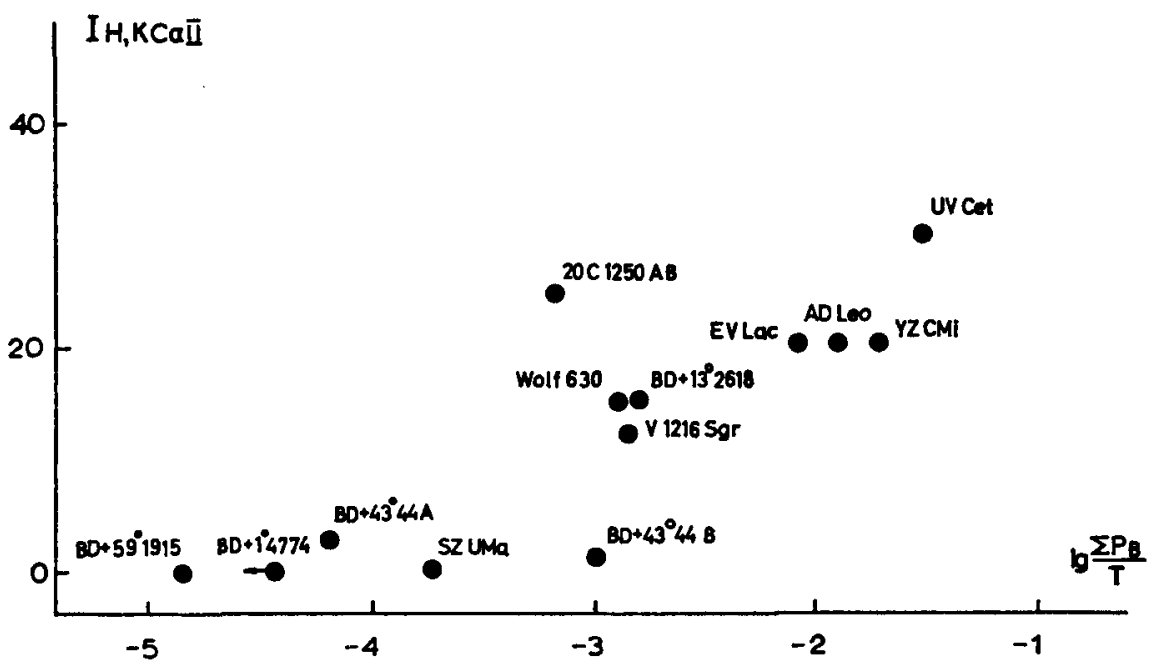

Fig. 10: Correlation between flare activity levels of stars and $\mathrm{Ca}$ emission intensities in their quiet state spectra.

Ref erences:

(1) R. E. GERSHBERG, P. F. CHUGAINOV, 1966, A. J. (USSR) 43, 1168; 1967, 44, 260.

(2) N. I. SHAKHOVSKAYA, 1969 , IBVS N339.

(3) E. K. ZAVOJSKI J, M. M. BUTSLOV, S D. FANCHENKO, 1970, Prriroda N8, 10.

(4) R. E. GERSHBERG, Izvestiya Crimean Astrophys. Obs. 45.

(5) W. E. KUNKEL, 1967, Dissertation. Austin.

(6) P. F. CHUGAINOV, Izvestiya Crimean Astrophys. Obs. 44.

(7) V. G. BUSLAVSKIJ, A. B. SEVERNY, 1969, „Stars, nebulae, galaxies“. Erevan, p. 129.

(8) Z. Svestka, M. Kopecki, M. Blaha, 1962, BAC 13, 37.

(9) V. G. BANIN, 1965, Tzvestiya Crimean Astrophys. Obs. 33, 118.

(10) R. E. GERSHBERG, N. I. SHAKHOVSKAYA, 1971, A. J. (USSR) 48.

(11) R. E. GERSHBERG, S. A. KAPLAN, Izvestiya Crimean Astrophys. Obs. 44.

(12) A. A. BOYARCHUK, 1966, Izvestiya Crimean Astrophys. Obs. 35, 45.

(13) O. C. WILSON, 1961, PASP 73, 15.

(14) R. N. THOMAS, R. G. ATHEY, 1961, Physics of the solar chromosphere. London.

(15) F. D. KAHN, 1969, Nature 222, 1130.

(16) R. E. GERSHBERG, 1970, Astrofizika 6, 191.

(17) R. E. GERSHBERG, Izvestiya Crimean Astrophys. Obs. 43.

(18) M. E. BOYARCHUK, Izvestiya Crimean Astrophys. Obs. 46.

(19) N. I. SHAKHOVSKAYA, Tzvestiya Crimean Astrophys. Obs. 45.

(20) N. I. SHAKHOVSKAYA, Izvestiya Crimean Astrophys. Obs. 47.

(21) P. F. CHUGAINOV, Izvestiya Crimean Astrophys. Obs. 46.

(22) P. F. CHUGAINOV, N. I. SHAKHOVSKAYA, 1971, IBVS N552.

(23) N. I. SHAKHOVSKAYA, K. L. MASLENNIKOV, 1970, IBVS N487.

(24) W. GLIESE, 1969, Veröff. Astron. Rechen-Institut Heidelberg N22.

(25) O. J. EGGEN, 1968, Aph. J. Suppl. N142, 77.

(26) A. H. JOY, R. E. WILSON, 1049, Aph. J. 109, 231.

\section{Discussion to the paper of GERSHBERG and SHAKHOVSKAYA}

SCHUMANN: What is the spectral resolution of the spectrographic equipment?

SCHÖFFEL: The final resolution is about $10 \AA$ for $340 \AA / \mathrm{mm}$. For dispersions 150,75 and $24 \mathrm{~A} / \mathrm{mm}$ the resolution is $4,2,0.6 \AA$ respectively. 